\title{
Unimodular waveform design for MIMO radar transmit beamforming
}

\author{
Hongtao $\mathrm{Li}^{1,2}$, Cheng Chen ${ }^{1}$ and Xiaohua $\mathrm{Zhu}^{1}$ \\ ${ }^{1}$ Nanjing University of Science and Technology, Nanjing, China \\ ${ }^{2}$ CETC Key laboratory of aerospace information applications, Shijiazhuang, China
}

\begin{abstract}
In this paper, we consider unimodular waveform optimization for MIMO radar transmit beamforming with prior information on the directions of target and interferences. A phase-only conjugate gradient method is presented to optimize the unimodular waveform by maximizing the ratio of power at the target location to the power at the interference locations. Simulations show that the transmit beampattern obtained from the optimized waveform achieves a coherent gain of the target location while minimizing the radiation powers around the interference locations.
\end{abstract}

\section{Introduction}

Compared to the traditional phased-array radar, the multiple-input multiple-output (MIMO) radar allows each transmit array element to transmit arbitrary waveform [1-5]. By exploiting waveform diversity, we can design the transmit beampattern for colocated MIMO radar flexibly. Traditionally, the waveforms transmitted from each antenna are designed to maximize the radiation power of the target location or to match a given transmit beampattern [6-9]. However, these methods fail to meet the demand for designing transmit waveforms with a beampttern which places nulls at the interference locations. Recently, a phase-only variable metric method (POVMM) is proposed to design waveforms for MIMO radar transmit beamforming with nulls in the directions of the interferences [3]. However, optimizing transmit waveforms, which match the beampattern with a significant gain at the target location and nulls at the interference locations, still remains an unsolved problem. In this paper, a phase-only conjugate gradient method is proposed to optimize unimodular waveforms to obtain such a beampattern by maximizing the ratio of power at the target location to the power at the locations of interferences.

\section{Signal model}

Consider a MIMO radar system with a uniform linear array (ULA) of $M$ transmit antennas. Let the waveform sequence with sample length $L$ transmitted from the $i$ th transmit antenna be $s_{i} \in J^{L \times 1}$, where $i \in\{1,2, \ldots, M\}$. Then the covariance matrix of the transmit signal can be given by $\boldsymbol{R}=\boldsymbol{S} \boldsymbol{S}^{\mathrm{H}} / L$, where $\boldsymbol{S}=\left[\boldsymbol{s}_{1}, \boldsymbol{s}_{2}, \ldots, \boldsymbol{s}_{M}\right]^{\mathrm{T}}$ denotes the transmit waveform matrix. Suppose the inter element spacing between two adjacent antennas is half of the carrier wavelength, and the transmit steering vector is given by:

$$
\boldsymbol{a}(\theta)=\frac{1}{\sqrt{M}}\left[1, e^{-j \pi \sin \theta}, \ldots, e^{-j \pi(M-1) \sin \theta}\right]^{\mathrm{T}}
$$

The radiation power at location $\theta$ can be written as:

$$
\begin{aligned}
P(\theta) & =\boldsymbol{a}^{\mathrm{T}}(\theta) \boldsymbol{R} \boldsymbol{a}^{*}(\theta) \\
& =\boldsymbol{a}^{\mathrm{T}}(\theta) \boldsymbol{S} \boldsymbol{S}^{\mathrm{H}} \boldsymbol{a}^{*}(\theta) / L
\end{aligned}
$$

Let $s$ be a $L M \times 1$ vector obtained from stacking the columns of transmit waveform matrix $\boldsymbol{S}$. Then, Eq. (2) can be rewritten as

$$
\begin{aligned}
P(\theta) & =\boldsymbol{s}^{\mathrm{H}}\left(\boldsymbol{I}_{L} \otimes \boldsymbol{a}^{*}(\theta)\right)\left(\boldsymbol{I}_{L} \otimes \boldsymbol{a}^{\mathrm{T}}(\theta)\right) \boldsymbol{s} / L \\
& =\boldsymbol{s}^{\mathrm{H}}\left(\boldsymbol{I}_{L} \otimes \boldsymbol{a}^{*}(\theta) \boldsymbol{a}^{\mathrm{T}}(\theta)\right) \boldsymbol{s} / L \\
& =\boldsymbol{s}^{\mathrm{H}} \boldsymbol{A}(\theta) \boldsymbol{s} / L
\end{aligned}
$$

where

$$
\boldsymbol{A}(\theta)=\boldsymbol{I}_{L} \otimes \boldsymbol{a}^{*}(\theta) \boldsymbol{a}^{\mathrm{T}}(\theta)
$$

Where $\boldsymbol{I}_{L}$ is a $L \times L$ identity matrix and $\otimes$ denotes the Kronecker product operator.

In the scenario of interest, a target is located at the angle $\theta_{t}$ and $K$ interferences are located at the angles of $\left\{\theta_{1}, \theta_{2}, \ldots, \theta_{K}\right\}$. To design a beampattern which concentrates the transmit power at the target location and places nulls at the interference locations, we formulate the constrained optimization problem as follows: 


$$
\begin{aligned}
& J=\max _{\boldsymbol{s}} \frac{\boldsymbol{s}^{\mathrm{H}} \boldsymbol{A}\left(\theta_{t}\right) \boldsymbol{s}}{\sum_{k=1}^{K} \boldsymbol{s}^{\mathrm{H}} \boldsymbol{A}\left(\theta_{k}\right) \boldsymbol{s}} \\
& \text { s.t. }|\boldsymbol{s}(i)|=1, i=1,2, \ldots, L M
\end{aligned}
$$

where the constant modulus constraint is considered due to the limited dynamic range of the radar amplifiers [10].

\section{Optimal unimodular waveform design}

Since $\boldsymbol{s}$ is an unimodular sequence, it can be written as:

$$
\boldsymbol{s}=\left[e^{j \varphi_{1}}, e^{j \varphi_{2}}, \ldots, e^{j \varphi_{L M}}\right]^{\mathrm{T}}
$$

Therefore, the waveform optimization problem is equivalent to the phase-only optimization problem and a conjugate gradient method can be used to obtain the optimal waveform.

To compute the gradient of the phase of the waveform, we consider extracting the first-order term of Taylor series expansion of $J$ [3, 11]. Let $\boldsymbol{\Delta}=\operatorname{Diag}\left(\delta_{1}, \delta_{2}, \ldots, \delta_{L M}\right)$ be a diagonal matrix where $\delta_{i}(i=1,2, \ldots, L M)$ is the small perturbation of the phase code $\varphi_{i}$. After the unimodular sequence is perturbed as $s \rightarrow e^{j u} s$, the Taylor series expansion of $J\left(e^{j x \Delta} s\right)$ is expressed as follows:

$$
J\left(e^{j x \boldsymbol{\Delta}} \boldsymbol{s}\right)=J(\boldsymbol{s})+x \mathrm{~d} J(\boldsymbol{s})(\boldsymbol{\Delta})+\cdots
$$

where $x$ is a scalar and

$$
\mathrm{d} J(\boldsymbol{s})(\boldsymbol{\Delta})=\left.\frac{\mathrm{d}}{\mathrm{d} x}\right|_{x=0} J\left(e^{j x \boldsymbol{\Delta}} \boldsymbol{s}\right)=\sum_{1 \leq i \leq L M} \frac{\partial J}{\partial \varphi_{i}} \delta_{i}
$$

Define

$$
N\left(e^{j x \boldsymbol{s}} \boldsymbol{s}\right)=\boldsymbol{s}^{\mathrm{H}} e^{-j x \boldsymbol{A}} \boldsymbol{A}\left(\theta_{t}\right) e^{j x \boldsymbol{s}} \boldsymbol{s}
$$

and

$$
D\left(e^{j x \Delta} \boldsymbol{s}\right)=\boldsymbol{s}^{\mathrm{H}} e^{-j x \Delta} \sum_{k=1}^{K} \boldsymbol{A}\left(\theta_{k}\right) e^{j x \Delta} \boldsymbol{s}
$$

The Taylor series expansion of $N\left(e^{j x \Delta} \boldsymbol{s}\right)$ and $1 / D\left(e^{j x \Delta} s\right)$ are respectively given by:

$$
N\left(e^{j x \boldsymbol{A}} \boldsymbol{s}\right)=\boldsymbol{s}^{\mathrm{H}}\left(\boldsymbol{A}\left(\theta_{t}\right)-j\left[\boldsymbol{\Delta}, \boldsymbol{A}\left(\theta_{t}\right)\right]+\cdots\right) \boldsymbol{s}
$$

$$
\begin{aligned}
1 / D\left(e^{j x \boldsymbol{\Delta}} \boldsymbol{s}\right)= & 1 /\left(\boldsymbol{s}^{\mathrm{H}} \sum_{k=1}^{K} \boldsymbol{A}\left(\theta_{k}\right) \boldsymbol{s}\right)+\boldsymbol{s}^{\mathrm{H}}\left(j\left[\boldsymbol{\Delta}, \sum_{k=1}^{K} \boldsymbol{A}\left(\theta_{k}\right)\right]\right. \\
& \left.+\frac{1}{2}\left[\boldsymbol{\Delta},\left[\boldsymbol{\Delta}, \sum_{k=1}^{K} \boldsymbol{A}\left(\theta_{k}\right)\right]\right]\right) \boldsymbol{s} /\left(\boldsymbol{s}^{\mathrm{H}} \sum_{k=1}^{K} \boldsymbol{A}\left(\theta_{k}\right) \boldsymbol{s}\right)^{2}+\cdots
\end{aligned}
$$

where the Lie bracket in Eqs. (11) and (12) is calculated as $[\boldsymbol{A}, \boldsymbol{B}]=\boldsymbol{A} \boldsymbol{B}-\boldsymbol{B} \boldsymbol{A}$. Then the first-order term $\mathrm{d} J(s)(\Delta)$ can be computed from the product of the Taylor series expansion of $N\left(e^{j x \Delta} s\right)$ and $1 / D\left(e^{j x \Delta} s\right)$, as follows:

$$
\mathrm{d} J(\boldsymbol{s})(\boldsymbol{\Delta})=\frac{-j}{\boldsymbol{s}^{\mathrm{H}} \sum_{k=1}^{K} \boldsymbol{A}\left(\theta_{k}\right) \boldsymbol{s}} \operatorname{Tr}\left\{\left(\boldsymbol{\Delta}\left[\boldsymbol{A}\left(\theta_{t}\right)-J(\boldsymbol{s}) \sum_{k=1}^{K} \boldsymbol{A}\left(\theta_{k}\right), \boldsymbol{s} \boldsymbol{s}^{\mathrm{H}}\right]\right\}\right.
$$

where $\operatorname{Tr}(\cdot)$ denotes the trace of a matrix.

By comparing Eqs. (8) and (13), the phase-only gradient of $J$ is given by the follow expression:

$$
\nabla \boldsymbol{J}=\frac{1}{\boldsymbol{s}^{\mathrm{H}} \sum_{k=1}^{K} \boldsymbol{A}\left(\theta_{k}\right) \boldsymbol{s}} \operatorname{Im}\left(\operatorname{diag}\left[\boldsymbol{A}\left(\theta_{t}\right)-J(\boldsymbol{s}) \sum_{k=1}^{K} \boldsymbol{A}\left(\theta_{k}\right), \boldsymbol{s} \boldsymbol{s}^{\mathrm{H}}\right]\right)
$$

where $\operatorname{Im}(\cdot)$ and $\operatorname{diag}(\cdot)$ denote imaginary part and diagonal part of a matrix respectively.

As the phase-only gradient $\nabla J$ is given by Eq. (14), a conjugate gradient method [12] can be used to obtain the optimal waveform. Let $\boldsymbol{\varphi}$ be a $L M \times 1$ vector of the phase code $\varphi_{i}(i=1,2, \ldots, L M)$, and the basic steps of the algorithm is summarized as follow:

a) For $i=0$, select an initial phase code vector $\varphi_{0}$ for transmit waveform, compute $\boldsymbol{g}_{0}=\boldsymbol{h}_{0}=\nabla J\left(\boldsymbol{\varphi}_{0}\right)$.

b) Let $i=i+1$, for all $x_{i} \geq 0$, find $t_{i}$ such that $J\left(\boldsymbol{\varphi}_{i-1}+x_{i} \boldsymbol{h}_{i-1}\right) \geq J\left(\boldsymbol{\varphi}_{i-1}\right)$.

c) Let $\boldsymbol{\varphi}_{i}=\boldsymbol{\varphi}_{i-1}+x_{i} \boldsymbol{h}_{i-1}$.

d) Compute $\boldsymbol{g}_{i}=\nabla J\left(\boldsymbol{\varphi}_{i}\right) \quad, \quad \boldsymbol{h}_{i}=\boldsymbol{g}_{i}+a_{i-1} \boldsymbol{h}_{i-1}$, $a_{i-1}=\frac{\left(\boldsymbol{g}_{i}-\boldsymbol{g}_{i-1}\right)^{\mathrm{T}} \boldsymbol{g}_{i}}{\left\|\boldsymbol{g}_{i-1}\right\|_{2}^{2}}$.

e) If $\left|J\left(\varphi_{i}\right)-J\left(\varphi_{i-1}\right)\right| \leq \varepsilon$, where $\varepsilon \geq 0$ is a parameter to control the convergence, let $\varphi_{i}$ be the optimal phase code vector; otherwise, go to step b).

\section{Simulation}

In this section, the performance of transmit beampattern obtained from the proposed method is compared to the 
method in [3]. We consider two cases which have different interference distributions. In case 1, the interference locations are discretely distributed at $\left\{-40^{\circ},-5^{\circ}, 45^{\circ}, 50^{\circ}\right\}$; in case 2 , the interferences are continuously distributed between $-25^{\circ}$ and $-10^{\circ}$. Suppose the target location of interest is $20^{\circ}$ and the transmit array consists of 16 and 32 antennas.

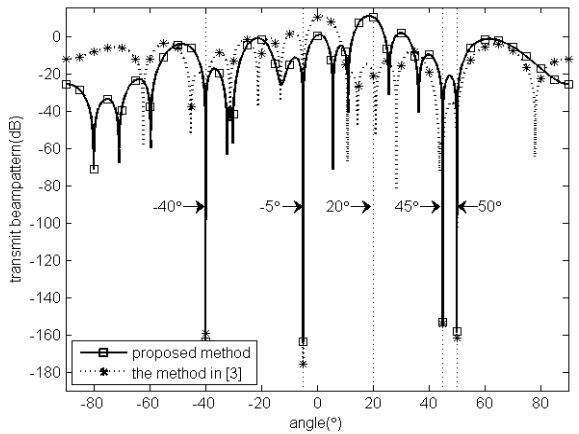

$a$ Transmit beampatterns with 16 transmit array elements

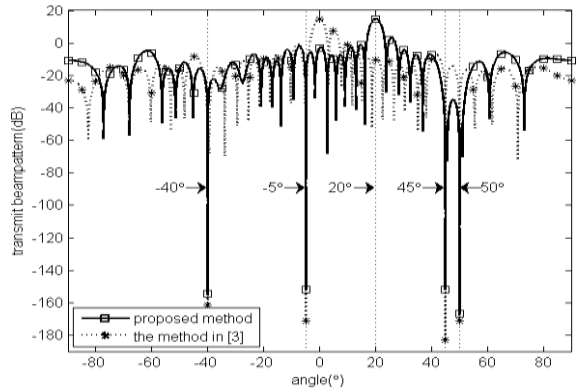

$b$ Transmit beampatterns with 32 transmit array elements

Fig 1. Comparisons of transmit beampattern

Fig. 1 and Fig. 2 depict the transmit beampatterns for the interferences of discrete distribution and continuous distribution respectively. Among the four plots, the proposed method can achieve a coherent gain at the target location, while the method in [3] forms a gain at arbitrary locations. In Fig. 1a and 1b, the null depths of at least $150 \mathrm{~dB}$ are obtained from both the methods with 16 and 32 transmit array elements. It seems that the number of the array elements has little effect on null depths obtained from the proposed method and the method in [3] shows better performance in interference suppression when the number of the array element is 32. In Fig. 2, the method in [3] shows almost $8 \mathrm{~dB}$ deeper null depth than the proposed method both in the case of 16 and 32 transmit array elements, without concentrating the power in the target direction. We can see that a transmit beampattern with 32 transmit array elements shows better null performance than the one with 16 elements. This is due to the fact that more degrees of freedom provided by 32 transmit array elements are used to reject interferences.

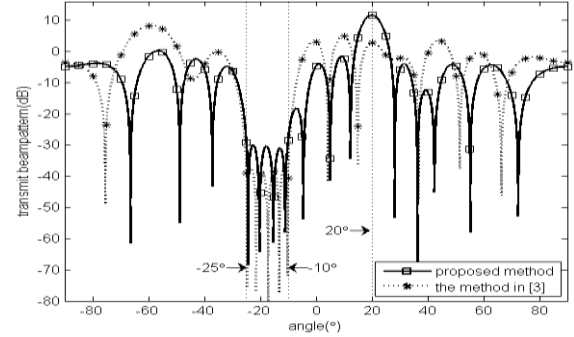

$a$ Transmit beampatterns with 16 transmit array elements

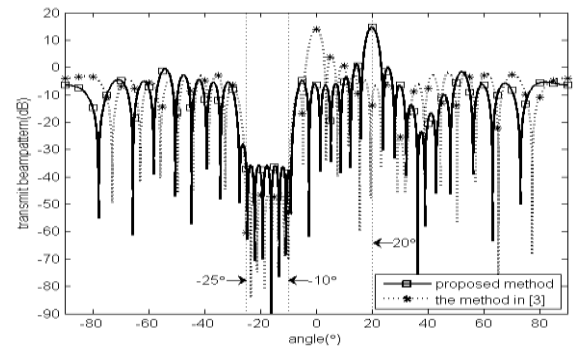

$b$ Transmit beampatterns with 32 transmit array elements

Fig 2. Comparisons of transmit beampattern

\section{Conclusion}

In this paper, unimodular waveforms have been optimized for MIMO radar transmit beamforming by maximizing the ratio of power at the target location to the power at the interference locations. Numerical results show that the beampatterns can achieve a coherent gain in the target location while forming nulls in the interference locations.

\section{Conflict of interests}

The authors declare that there is no conflict of interests regarding the publication of this paper.

\section{Acknowledgment}

This work was supported by the National Natural Science Foundation of China (Grant no. 61401204), Postdoctoral Science Foundation of Jiangsu Province (Grant no. 1501104C), Technology Research and Development Program of Jiangsu Province (Grant no. BY2015004-03), China Postdoctoral Science Foundation (Grant no. 155676), and the Fundamental Research Funds for the Central Universities (Grant no. 30916011319).

\section{References}

1. Jian, L., Stoica, P. 'MIMO radar with colocated antennas', IEEE Signal Porcess. Mag., (2007), 24, (5), pp. 106-114, doi: 10.1109/MSP.2007.904812

2. Roberts W., He H., Stoica, P., and Jian, L.: 'Probing waveform synthesis and receiver filter design', IEEE Signal Porcess. Mag., (2010), 27, (4), pp. 99-112, doi: 10.1109/TSP.2010.936724 
3. Lilin, G., Hai, D., and Himed, B.: 'Waveform optimization for transmit beamforming with MIMO radar antenna arrays', IEEE Trans. Antennas and Propagation, (2015), 63, (2), pp. 543-552, doi: 10.1109/TAP.2014.2382637

4. Zhang, X., He, Z., Rayman-Bacchus, L., and Yan, J.: 'MIMO radar transmit beampattern matching design', IEEE Trans. Signal Porcess., (2015), 63, (8), pp. 2049-2056, doi: 10.1109/TSP.2015.2398841

5. Jardak, S., Ahmed, S., and Alouini, M., S.: 'Generation of correlated finite alphabet waveforms using gaussian random variables', IEEE Trans. Signal Porcess., (2014), 62, (17), pp. 4587-4596, doi: 10.1109/TSP.2014.2339800

6. Ahmed, S., Thompson, J. S., Petillot, Y., R., and Mulgrew, B.: 'Finite alphabet constant-envelope waveform design for MIMO radar', IEEE Trans. Signal Porcess., (2011), 59, (11), pp. 5326-5337, doi: 10.1109/TSP.2011.2163067

7. Stoica, P., Jian, L., and Yao, X.: 'On probing signal design for MIMO radar', IEEE Trans. Signal Porcess., (2007), 55, (8), pp. 4151-4161, doi: 10.1109/TSP.2007.894398
8. Stoica, P., Jian, L., and Xumin, Z.: 'Waveform synthesis for diversity-based transmit beampattern design', IEEE Trans. Signal Porcess., (2008), 56, (6), pp. 2593-2598, doi: 10.1109/TSP.2007.916139

9. Xu, H., Blum, R., S., Wang, J. and Yuan J.: 'Colocated MIMO radar waveform design for transmit beampattern formation', IEEE Trans. Aerosp. Electron. Syst., (2015), 51, (2), pp. 15581568, doi: 10.1109/TAES.2014.140249

10. Guolong, C., Hongbin, L., and Rangaswamy, M.: 'MIMO radar waveform design with constant modulus and similarity constraints', IEEE Trans. Signal Porcess., (2014), 62, (2), pp. 343-353, doi: 10.1109/TSP.2013.2288086

11. Zhang, J.D., Zhu, X.H., and Wang, H.Q.: 'Adaptive radar phase-coded waveform design', Electron. Lett., (2009), 45, (20), pp. 1052-1053, doi: 10.1049/el.2009.1099

12. Conjugate gradient method [online]. Available: http://en.wikipedia.org/wiki/ conjugate_gradient_method 